\title{
Epigenetically Reprogrammed Methylation as a Gifted Potential Cancer Biomarker
}

\author{
Seyed Mohammad Kazem Aghamir, Maryam Aghaii and Fatemeh Khatami* \\ Urology Research Center, Tehran University of Medical Sciences, Iran
}

*Corresponding author: Fatemeh Khatami, Urology Research Center, Sina Hospital, Hassan Abad Square, Tehran, Iran

\section{Introduction}

Ontrary to genetic changes of DNA molecule which change exactly the sequence of DNA and are not reversible, the DNA methylation involves in addition of a methyl group to cytosine nucleotide to control genes expression which is reversible $[1,2]$. Methylation is unique change that consequence in different gene expression pattern and finally may trigger the onset of diseases like cancer [3]. Epigenetic reprogramming in cancer cells represents a unique methylation landscape concerning the net loss of global DNA methylation simultaneously with an increase in the levels of in CpGs islands. So, cancer epigenetically reprogrammed methylation landscape (i.e., Methylscape) can be a common feature exhibited by most cancer types and therefore can be a universal cancer biomarker. The DNA methylation in molecular level change the gene expression pattern of the cell but methylation can change the physicochemical properties of DNA polymer in solution including DNA structure and its affinity as well.

It is shown by Abu Ali Ibn Sina and his colleagues examined the consequence of levels and genomic distribution of methylcytosines on the physicochemical properties of DNA to sense the Methylscape biomarker [4]. They found that DNA polymeric behavior is powerfully affected by differential patterning of methylcytosine resulting in fundamental differences in DNA solvation and DNAgold affinity as the discriminative biomarker between cancerous and normal genomes. They use the Methylscape differences to develop simple, greatly sensitive and selective electrochemical or colorimetric one-step assays for the detection of cancer.

In mammalian genomes, DNA methyltransferases (DNMTs) duty is transferring the methyl group from S-adenosylmethionine to cytosine at $\mathrm{CpG}$ dinucleotides (CpG islands) [5]. The majority of the methylation happens through DNA duplication in the S-phase of the cell cycle, and is the supreme rich form of post-replicative DNA alteration of eukaryotic organisms [6,7]. DNA methylation cause the $180^{\circ}$ flip out of the DNA backbone into an active-site pocket of the enzyme where methylation of cytosine takes place [8]. In fact, well methylated DNA (hypermethylated) is classically coupled with inactive genes, whereas methylation depletion (hypomethylation) can be observed in active genes.

Actually it is confirmed that DNA segments containing methylated Cytosine like something which is happening in cancer cells, are very stiff and hard to bend, and present an inferior tendency to circularize or form nucleosomes by wrapping around histones and can be used as the discriminative universal cancer biomarker [4]. There is a big hope that in the near future by using some physical properties of methylated DNA in comparison to nonmethylated DNA the diagnostic kits will be approved with no advanced complicated molecular technologies.

\section{References}

1. Schübeler D (2015) Function and information content of DNA methylation. Nature 517(7534): 321-326

2. Smith Z D, A Meissner (2013) DNA methylation: roles in mammalian development. Nature Reviews Genetics 14(3): 204-210.

3. Suzuki M M, A Bird (2008) DNA methylation landscapes: provocative insights from epigenomics. Nature Reviews Genetics 9(6): 465-476.

4. Sina A A I (2018) Epigenetically reprogrammed methylation landscape drives the DNA self-assembly and serves as a universal cancer biomarker. Nature communications 9(1): 4915.

5. Goll M G and T H Bestor (2005) Eukaryotic cytosine methyltransferases. Annu Rev Biochem 74: 481-514.

6. Leonhardt $\mathrm{H}$ (1992) A targeting sequence directs DNA methyltransferase to sites of DNA replication in mammalian nuclei. Cell 71(5): 865-873.

7. Clark T A ( 2011) Characterization of DNA methyltransferase specificities using single-molecule, real-time DNA sequencing. Nucleic acids research 40(4): e29

8. Pérez, A et al. (2012) Impact of methylation on the physical properties of DNA. Biophysical journal 102(9): 2140-2148. 
(c) (i) This work is licensed under Creative

To Submit Your Article Click Here: $\quad$ Submit Article

DOI: $10.32474 /$ OAJOM.2019.03.000156

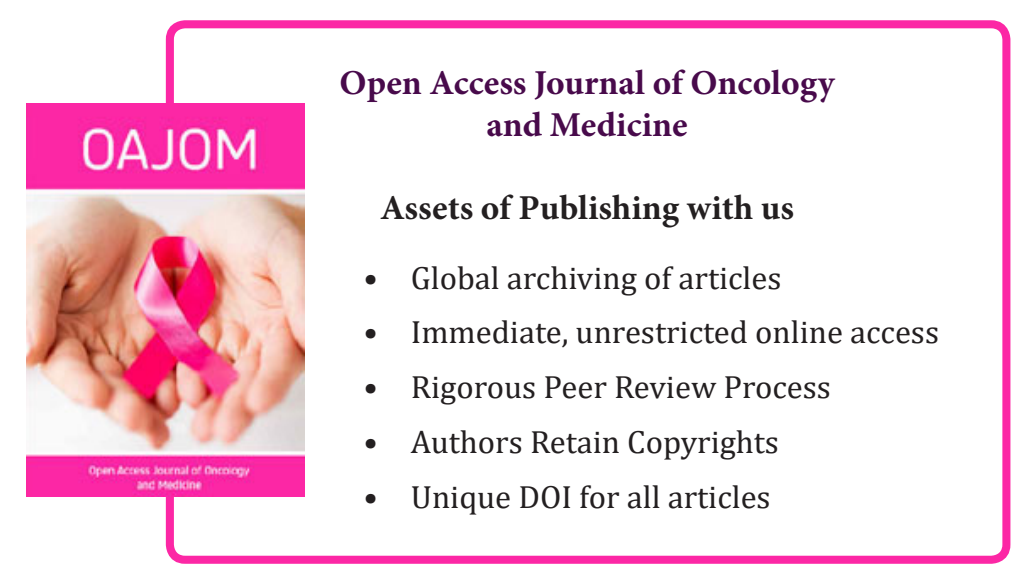

